Theoretical Physics, Astrophysics and Cosmology

Vol. 3, No 3, p. 16 - 35, v1, 29 August 2008 Online: TPAC: 2798-011 v2, 18 September 2012
ISSN 1819-9135; ISSN 1991-3919 (e)

(C) 2008, 2012 CTPA. All rights reserved. DOI: $10.9751 /$ TPAC.2798-011

\title{
Non-dissipative diffusion in classical and quantum systems
}

\author{
Zahid Zakir
}

\begin{abstract}
The theory of non-dissipative diffusion is constructed on an example of diffusion of a light particle in a dilute medium of heavy particles and it is shown that in low dissipative systems there are specific effects of non-dissipativity similar to quantum effects. In a non-dissipativity region mean energy of the light particle is conserved and processes are described by two non-linear diffusion equations with forward and backward time derivatives. Then these two diffusion equations are linearized and give one linear Schrödinger equation for complex amplitudes of probability. As a result, in the non-dissipative classical diffusion should be added probability amplitudes and there holds the superposition principle for these amplitudes. A mean square length of free passage and a mean square momentum define an elementary phase volume and a diffusion constant and they obey the uncertainty relations. It is shown that the formalism of quantum mechanics describes the classical non-dissipative diffusion with a homogeneous diffusion constant and that quantum mechanics is only a particular case when the elementary phase volume of free passage is universal and equal to the Planck constant.
\end{abstract}

PACS: 02.50.Ey, 03.65.Ta, 05.40.Jc,

Key words: stochastic processes, quantum mechanics, Brownian motion

\section{Content}

Introduction

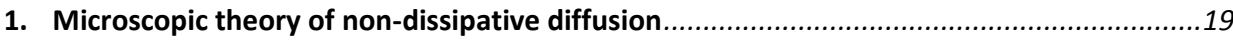

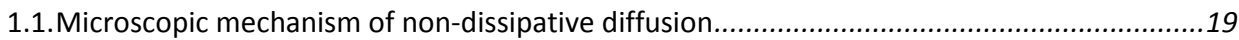

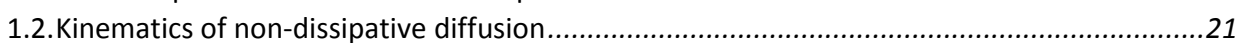

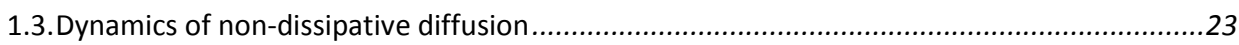

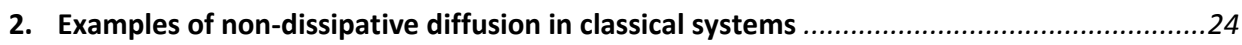

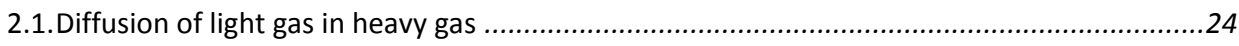

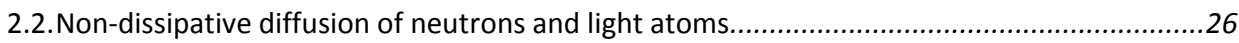

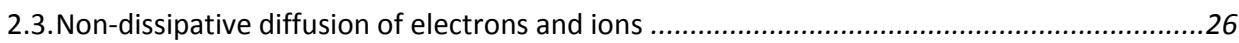

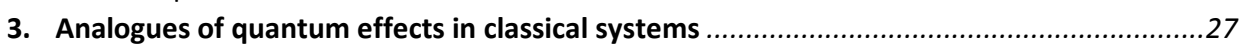

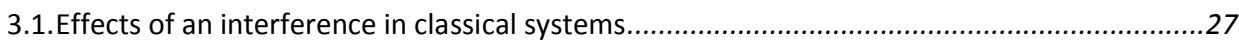

3.2. Discrete energy levels of oscillator and the uncertainty relation .......................................28

3.3. Discrete energy levels and discrete angular momenta of classical systems..........................28

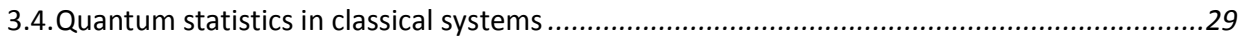

4. Quantum mechanics as the theory of non-dissipative diffusion .............................................

4.1. Insufficiency of standard interpretations of quantum mechanics............................................30

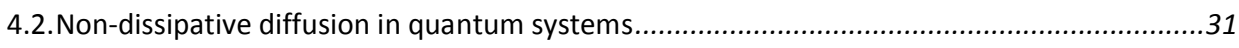

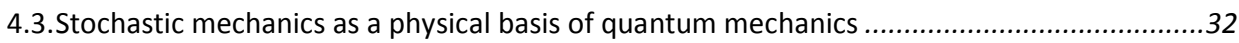

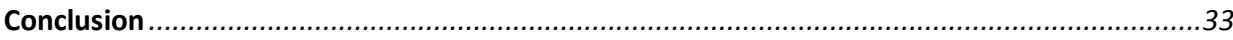

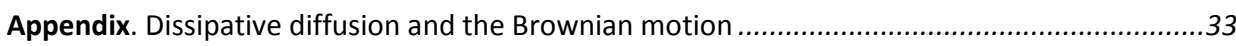

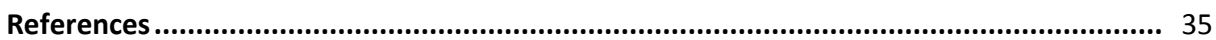

${ }^{1}$ Centre for Theoretical Physics and Astrophyics, Tashkent, Uzbekistan; zahidzakir@theor-phys.org 


\section{Introduction}

A microscopic mechanism of usual dissipative diffusion is based on a model of a random walk of a large heavy particle in a medium of many chaotically moving small and light particles [1], where the role of dissipation of energy of the diffusing particle is essential.

In the theories of random processes and condensed states approximately non-dissipative diffusion has been considered simply as a particular case of dissipative diffusion since it was considered that low dissipation does not lead to essentially new effects.

In the present paper diffusion in classical systems with low dissipation of energy is considered and it is shown that a mechanism of such diffusion essentially differs on the mechanism of dissipative diffusion and, at first approximation when we neglect by dissipation, there are qualitatively new effects in such systems. It follows from the fact that a microscopic mechanism of non-dissipative diffusion is based on model of a random walk of a small light particle in dilute medium of many chaotically moving large heavy particles. On a path of free passage a trajectory of a light particle is smooth and at any collision it collides only with a single heavy particle of the medium. Since these collisions elastic and recoil is small, mean energy of the light particle is conserved throughout large number of collisions and in this region of nondissipativity it is possible to consider diffusion approximately as non-dissipative one.

The existence of a path of free passage, where trajectory is smooth, and also statistically reversibility of the process dye to conservation of mean energy in the ensemble of diffusing particles, lead to two symmetric in time non-linear equations for a diffusional flow. These equations are linearized at transition to one complex probability amplitude. As a result, a probability addition law changes and it will appear that at the non-dissipative diffusion the probability amplitudes addition law as in quantum mechanics takes place.

This fact makes the theories of non-dissipative diffusion of special interest since still by Fényes [2] and Nelson [3] discovered that quantum mechanics in fact is a theory of a non-dissipative diffusion in the physical vacuum of a classical particle of mass $m$ with a fixed diffusion constant $v=\hbar / 2 m$.

Let's notice that the concept of non-dissipative diffusion, of course, should be considered as an idealization, like concepts of ideal gas or a point particle. In a mixture of two ideal gases diffusion of gas of heavy atoms in gas of light atoms is an elementary model of dissipative diffusion whereas in opposite case of diffusion of light gas in heavy gas [1] the effects of non-dissipative diffusion take place as a first approximation.

First theory based on non-dissipative diffusion has appeared only in connection with stochastic treatment of quantum mechanics [2-4]. Nelson derived the Schrödinger equation and the probability amplitudes addition law from the hypothesis about a non-dissipative Brownian motion of classical particles in physical vacuum. This approach, named as stochastic mechanics, in fact solved the problem of 
interpretation of quantum mechanics and has been developed in many publications [4].

Nevertheless, both the stochastic treatment of quantum mechanics and the discovered kinematics and dynamics of non-dissipative diffusion have not been widely accepted and did not become a part of the standard paradigm. It is related mainly with two problems at the formulation of this approach.

The first problem consisted in that the derivation of a formalism of nondissipative diffusion as a hybrid of former two ones (Einstein-Smoluchowski and Langevin-Ornstein-Uhlenbeck) looked artificial and initially contained an interior inconsistency. Nelson considered a medium with very large friction when coordinates of a Brownian particle are stochastic and hoped that in this highly dissipative medium a conservative (conserving mean energy) diffusion is possible, which in classical systems, obviously, is unrealizable. Therefore long time it failed to indicate an example of non-dissipative diffusion in classical systems where it would be possible to show and study unusual properties of this new kind of diffusion, declaring for quantum systems [4].

This problem of stochastic mechanics has been solved in 1987 when I presented [5] an example of non-dissipative diffusion in purely classical system. The interference effect in an ensemble of classical particles in two slit experiment at passing of an ensemble of light particles through a set of screens with many slits and shutters were considered. Collisions with rapidly opened and closed shutters lead to random changes of momentum and energy of the particles and elasticity of collisions leads to conservation of their mean energy. If Nelson tried to fall outside limits of the theory of dissipative diffusion by prolonging to consider a large particle in a dense medium with very large friction, on the contrary, in [5] it has been considered diffusion of a small particle in a dilute medium of heavy "particles" (shutters) when friction is negligible and full trajectory is collected on randomly changing pieces of classical trajectories.

Second weakness of stochastic mechanics was that it had not predicted new observable effects. This has been overcome in 1999 when I proposed a diffusional treatment of gravitation [6]. On the one hand, motion of a test particle in flat spacetime with a non-uniform diffusion constant is equivalent to its motion in curved spacetime with a uniform diffusion constant [4]. On the other hand, in fluctuating medium a concentration of many classical particles changes a state of medium and leads to inhomogeneity of the diffusion constant. As a result, test particles drift to a region with low diffusion constant and proper times of particles, depending on intensity of their fluctuations, are dilated.

The ability of stochastic mechanics to explain on unified basis both quantum phenomena and gravitation means that further it can be a basis for a more fundamental theory joining quantum mechanics and general relativity.

In the first part of the present paper the microscopic theory of non-dissipative diffusion in classical systems is formulated. In the second part examples of classical systems with non-dissipative diffusion are presented and analogues of quantum effects in them (quasi-quantum effects) are considered. In the third part the stochastic treatment of quantum phenomena is presented. 


\section{Microscopic theory of non-dissipative diffusion}

\subsection{Microscopic mechanism of non-dissipative diffusion}

A microscopic mechanism of diffusion process of one kind of particles in a medium of other kind of particles (atoms, molecules etc.), generally consists in random walking of diffusing particles at colliding with set of chaotically moving particles of the medium. There are two extreme cases in which diffusion processes qualitatively differ depending on a relation of masses of diffusing particles and particles of the medium.

A microscopic mechanism of dissipative diffusion is based on the model of a large and heavy particle moving in a medium of small and light particles. An elementary realization of such model is diffusion of ideal gas of heavy atoms in gas of light atoms [1]. There it is supposed that a diffusing particle: (1) is larger and heavier than particles of the medium; (2) in any small time interval collides with large number of particles of the medium. At such relation of sizes and masses of particles of the medium and the diffusing particle, very high number of collisions in any time interval, the dissipation of energy of the particle is large and inevitable.

A microscopic mechanism of non-dissipative diffusion is based on an opposite extreme case of diffusion of light particles of a small size in a dilute medium of heavy particles. Further considering only such classical systems, we will assume that:

a) a free pass length of particles in medium is larger than size of particles;

b) diffusing particle collides elastically with separate particles of medium;

c) diffusing particle's mass $m$ is much less than mass of particles of medium $M$, i.e. $m \ll M$.

From these properties of such systems follows that:

a) between collisions particles move along smooth classical paths;

b) loss of light particle's energy at such collisions is proportional $m / M$ and insufficient: $\delta E \sim(m / M) \ll E$, and thus in the first approximation we can neglect by dissipation in large enough number of collisions $N \sim(M / m) \gg 1$;

c) spatial size $L_{N D}$ and time interval $t_{N D}$ of a non-dissipativity region, where mean energy is approximately conserved, is inversely proportional to the mass ratio $L_{N D} \sim t_{N D} \sim M / m$.

At collisions with heavy particles of medium in a rest frame of each molecule mainly changes a direction of velocity of the light particle and a value of its velocity changes insufficiently. But in a laboratory frame, where the box with gas is rested, the fluctuations of diffusing particle's velocity will be of order of a thermal velocity of particles of medium and consequently:

$$
\delta v_{D}=V \sqrt{\frac{M}{m}}=\sqrt{\frac{6 k T}{m}},
$$

where $T$ is temperature of medium and $V$ is a value of mean velocity of the medium's particles.

Further we will take into account that values of fluctuations of coordinates of the particle before and after each collision are independent and consequently the 
stochastic component of the drift is the Markovian process when previous history of the particle "is forgotten". Therefore, initial kinetic energy of an ensemble of particles is only a single-time contribution which gradually dissipates whereas drift velocities and accelerations are defined mainly by regular and repeated changings of particle's momentum at action of an external field between the collisions.

Taking into account of the contribution of the external field simplifies due to that all trajectory of the diffusing particle consists of stick together smooth paths of free passage between collisions. This is first basic difference of the mechanism of non-dissipative diffusion from the mechanism of usual diffusion where such paths are absent and it is necessary to smooth them artificially.

Under these requirements the initial energy of light atoms is conserved throughout large enough number of collisions $n_{D} \sim M / m$. In lack of external forces this part of particle's trajectory is $n_{D} l_{D}$ ( $l_{D}$ is the free passage length) and corresponding time interval $t_{D}$ we will call as a region of non-dissipativity. Therefore the mean length of classical parts of the trajectory $\Delta r_{c l}$ is equal to the free passage length $l_{D}$ in the medium and the mean time interval of free passage is equal to $\Delta t_{c l}=\Delta r_{c l} / v_{D}$, where $v_{D}$ - a mean velocity of free passage of the particle.

In fact the energy received by a particle from external forces between collisions (on classical parts of trajectory), is partially used up for compensation of loss on the dissipation. In this case, when losses of mean energy of a particle on dissipation are partially compensated along a direction of acceleration of external force, we deal with the extended region of non-dissipativity along that direction. In such effectively non-dissipative systems where the external field fully compensates losses on dissipation, qualitative differences of non-dissipativity will be revealed in a pure form and in full volume of the system.

Thus, effective mean energy of the diffusing particle consisting of energy of thermal motion and mean energy, accumulated in an external field between collisions minus losses on dissipation, it is possible to consider as conserved one. Then the process is statistically reversible and there is symmetry under the time reversal. Therefore in following section a kinematics of the symmetric in time diffusion process already without these conditions and improvements of the microscopic mechanism of such diffusion will be considered.

Let a light particle starts to diffuse in a dilute medium of heavier particles. By averaging on ensemble of light particles in each moment $t$ a mean length $l_{D}$, a mean time $\tau_{D}$ and a mean velocity $v_{D}=l_{D} / \tau_{D}$ of free passage can be defined. The last one is related to a mean momentum and particle's kinetic energy as usual $p_{D}=m v_{D}, E_{D}=m v_{D}^{2} / 2$.

The ensemble of light particles is not under usual thermodynamic equilibrium with medium, but in a dynamical equilibrium when mean energy of light particles can essentially differ from energy of thermal motion and $l_{D}, \tau_{D}$ can depend on time and from external force also. 
Nevertheless, the existence of classical parts of a trajectory with the singled out mean characteristics $l_{D}, p_{D}$ is a basic difference of considered diffusion from usual diffusion. In particular, due to conservation of particle's mean energy, it is possible to introduce mean value of an abbreviated action function $S$ in the time interval $t^{\prime}-t=N \tau_{D}, N \gg 1$. It represents the sum over short classical parts of the trajectory:

$$
\Delta S=N S_{D}, \quad S_{D}=p_{D} l_{D},
$$

where $S_{D}$ is an elementary abbreviated action of the medium separating a region of classicity of trajectories from a region of stochasticity.

However, statistical mechanics deals with not by the action function along trajectories, but with a value of a phase volume $\Delta \Gamma=\Delta p \Delta x$ in which a particle is placed during a time slice $\Delta t$. In our case the particle at free passage time $\tau_{D}$ places in a sell of size equal to the free pass length $l_{D}$ and has the mean momentum $p_{D}=m l_{D} / \tau_{D}$. Then for such particle there is an elementary phase volume:

$$
\Delta \Gamma=p_{D} l_{D}=\frac{m l_{D}^{2}}{\tau_{D}}=\Gamma_{D},
$$

which coincides with the elementary abbreviated action $S_{D}$ and also separates the classicity region of trajectories on the stochasticity region.

Since $\Gamma_{D}$ plays such essential role, be as the elementary phase volume for the particles, it will be more natural to take it as primary quantity and to express through it other kinematical and dynamical characteristics of the system. In particular, from (3) and the definition of the diffusion constant $l_{D}^{2}=2 v \tau_{D}$ follows

$$
l_{D}^{2}=\frac{\Gamma_{D}}{m} \tau_{D}=2 v \tau_{D}, \quad v=\frac{\Gamma_{D}}{2 m},
$$

i.e. the diffusion constant $2 v$ in our system is in fact elementary phase volume $\Gamma_{D}$ for a unit mass particle.

\subsection{Kinematics of non-dissipative diffusion}

Let particle's coordinates are fixed through small time intervals $\Delta t$, equal to a mean pass time of the diffusing particle between collisions in points $\mathbf{x}(t-\Delta t), \mathbf{x}(t)$ and $\mathbf{x}(t+\Delta t)$, when its motion occurs on classical trajectories. All averaging are made not only on transition probabilities from an event $\mathbf{x}(t)$ to a set of events $\mathbf{x}(t+\Delta t)$ as in case of usual diffusion, but also on transition probabilities from a set of events $\mathbf{x}(t-\Delta t)$ to the event $\mathbf{x}(t)$.

Then the drift velocity is defined as:

$$
\mathbf{b}_{ \pm}(\mathbf{x}(t), t)=D_{ \pm} \mathbf{x}(t) \equiv \lim _{\Delta t \rightarrow 0+} E_{t} \frac{\mathbf{x}(t \pm \Delta t)-\mathbf{x}(t)}{ \pm \Delta t} .
$$


As a result, from two drift velocities $\mathbf{b}_{+}, \mathbf{b}_{-}$, obtained by an averaging over two sets of random processes, it is possible to combine a drift velocity $\mathbf{v}$ and a mean velocity of fluctuations $\mathbf{u}$ :

$$
\begin{array}{ll}
\mathbf{v}=\frac{1}{2}\left(\mathbf{b}_{+}+\mathbf{b}_{-}\right), & \mathbf{u}=\frac{1}{2}\left(\mathbf{b}_{+}-\mathbf{b}_{-}\right), \\
\mathbf{b}_{+}=\mathbf{v}+\mathbf{u}, & \mathbf{b}_{-}=\mathbf{v}-\mathbf{u} .
\end{array}
$$

Velocity $\mathbf{v}$ in a classical limit of a particle with the smooth trajectory transfers in usual velocity of Newtonian mechanics.

Particle's shifts then are described by the stochastic equations:

$$
d \mathbf{x}_{ \pm}(t)=\mathbf{b}_{ \pm}(\mathbf{x}(t), t) d t+d \mathbf{w}_{ \pm}(t),
$$

where $d \mathbf{w}_{ \pm}(t)$ is a random process with properties:

$$
\begin{aligned}
& \lim _{\Delta t \rightarrow 0+} E_{t}\left[\Delta \mathbf{w}_{ \pm}(t)\right]=0, \\
& \lim _{\Delta t \rightarrow 0+} E_{t}\left[\Delta w_{ \pm}^{i}(t) \Delta w_{ \pm}^{i}(t)\right]= \pm 2 v \delta^{i j} d t .
\end{aligned}
$$

Probability density $\rho(x, t)$ is normalized:

$$
\int \rho(\mathbf{x}(t), t) d x(t)=1,
$$

and satisfies the continuity equation:

$$
\partial \rho / \partial t=-\nabla \cdot\left(\mathbf{b}_{ \pm} \rho\right) \pm v \Delta \rho,
$$

Corresponding transition probabilities, defined at $t^{\prime \prime}>t>t^{\prime}$ as:

$$
\begin{gathered}
\rho(\mathbf{x}(t), t)=\int p_{-}\left[x(t), t ; x^{\prime}\left(t^{\prime}\right), t^{\prime}\right] \rho\left(x^{\prime}\left(t^{\prime}\right), t^{\prime}\right) d x\left(t^{\prime}\right), \\
\int d x^{\prime \prime}\left(t^{\prime \prime}\right) \rho\left(x^{\prime \prime}\left(t^{\prime \prime}\right), t^{\prime \prime}\right) p_{+}\left[x^{\prime \prime}\left(t^{\prime \prime}\right), t^{\prime \prime} ; x(t), t\right]=\rho(x(t), t),
\end{gathered}
$$

satisfy the equation:

$$
p_{ \pm}\left[x\left(t^{\prime \prime}\right), t^{\prime \prime} ; x^{\prime}\left(t^{\prime}\right), t^{\prime}\right]=\int p_{ \pm}\left[x\left(t^{\prime \prime}\right), t^{\prime \prime} ; x(t), t\right] d x(t) p_{ \pm}\left[x(t), t ; x^{\prime}\left(t^{\prime}\right), t^{\prime}\right] .
$$

Mean time derivatives from functions of coordinates and time $f(x(t), t)$, according to (5) and (8), are defined as:

$$
D_{ \pm} f(\mathbf{x}(t), t)=\left(\partial / \partial t+\mathbf{b}_{ \pm} \cdot \nabla \pm v \Delta\right) f(\mathbf{x}(t), t) .
$$

Combining two equations for a probability density (10), we obtain, on the one hand, the continuity equation:

$$
\frac{\partial \rho}{\partial t}+\nabla \cdot(\mathbf{v} \rho)=0
$$

where $\mathbf{v}(x(t), t)$ is entered. On the other hand, subtracting the equations (6) and (12), we have:

$$
\nabla \cdot(\mathbf{u} \rho)=v \nabla \cdot(\nabla \rho),
$$

and further we obtain expression of unsymmetrical on time (and fluctuating) part of the drift velocity $\mathbf{u}(\mathbf{x}(t), t)$ through $\rho$ and $v$ :

$$
\mathbf{u}=v \frac{\nabla \rho}{\rho}=v \nabla \ln \rho .
$$

A mean value of $\mathbf{u}$ is equal to zero: 


$$
\overline{\mathbf{u}}=\int \mathbf{u} \rho d x=0,
$$

and this fluctuating component of velocity disappears in the limit $v \rightarrow 0$.

Last property, together with (17), leads to the uncertainty relation (at $\overline{\mathbf{x}}=0$ ) for a fluctuating component of the momentum $\mathbf{p}_{u}=m \mathbf{u}$ :

$$
\sqrt{\overline{(m \mathbf{u})^{2} \cdot \overline{\mathbf{x}^{2}}}} \geq|\overline{m \mathbf{u} \cdot \mathbf{x}}|=m\left|\int \mathbf{u} \cdot \mathbf{x} \rho d x\right|=m v\left|\int(\nabla \rho) \cdot \mathbf{x} d x\right|=m v .
$$

Using the continuity equation (15), further we obtain the time evolution equation for $\mathbf{u}$ :

$$
\frac{\partial \mathbf{u}}{\partial t}=-v \nabla(\nabla \cdot \mathbf{v})-\nabla(\mathbf{v} \cdot \mathbf{u})
$$

\subsection{Dynamics of non-dissipative diffusion}

For transition to dynamics of non-dissipative diffusion we will introduce further mean drift acceleration of a particle $\mathbf{a}(\mathbf{x}(t), t)$, defined as symmetric on both directions of time and related to an external force $\mathbf{F}$, due to lack of frictional force, across Newton's laws:

$$
\mathbf{a}=\frac{1}{2}\left(D_{-} D_{+}+D_{+} D_{-}\right) \mathbf{x}(t)=\frac{1}{m} \mathbf{F} .
$$

This equation, expressed through velocities $\mathbf{v}$ and $\mathbf{u}$, takes a form:

$$
\frac{\partial \mathbf{v}}{\partial t}=\frac{1}{m} \mathbf{F}-(\mathbf{v} \cdot \nabla) \mathbf{v}+\frac{1}{2}(\mathbf{u} \cdot \nabla) \mathbf{u}+v \Delta \mathbf{u} .
$$

Since trajectory of a diffusing particle between collisions is classical, therefore, as well as in Hamiltonian dynamics, the drift component of the momentum $\mathbf{p}_{\mathbf{v}}=m \mathbf{v}$ can be represented as a gradient of some function of coordinates and time $S(x, t)$ :

$$
m \mathbf{v}(x, t)=\nabla S(x, t) .
$$

Then an equation of motion (22) and the continuity equation (15), with the account (17) and taking $\mathbf{F}=-\nabla V$, can be written in the form:

$$
\begin{gathered}
\frac{\partial S}{\partial t}+\left(\frac{(\nabla S)^{2}}{2 m}+V\right)-\frac{(2 m v)^{2}}{2 m} \frac{\Delta \sqrt{\rho}}{\sqrt{\rho}}=0, \\
\frac{\partial \rho}{\partial t}+\frac{1}{m} \nabla \cdot(\rho \nabla S)=0 .
\end{gathered}
$$

It is nothing but as the well-known "hydrodynamic" representation of the Schrödinger equation proposed by Madelung still in 1926.

The first equation differs from the Hamilton-Jacobi equation by only last, nonlinear term concerning the probability density. But the system of equations (24)-(25) admits a linearization by a canonical transformation from the pair of real-valued functions $\rho(x, t), S(x, t)$ to one a complex function $\psi(x, t)$ :

$$
\psi(x, t)=\sqrt{\rho(x, t)} \exp \left[\frac{i S(x, t)}{2 m v}\right] .
$$


Then two equations (24)-(25) will give one equation for complex "wave function" $\psi(x, t)$ :

$$
i(2 m v) \frac{\partial \psi}{\partial t}=H \psi
$$

where the Hamiltonian looks like:

$$
H=-\frac{(2 m v)^{2}}{2 m} \Delta+V
$$

It is a Schrödinger equation, linear under $\psi(x, t)$ and consequently the principle of superposition for its solutions takes place:

$$
\psi=c_{1} \psi_{1}+c_{2} \psi_{2} .
$$

Considering the relation $\rho=|\psi|^{2}$, we see that at classical non-dissipative diffusion holds the probability amplitude addition law. The physical sense of a wave behavior consists in periodic repetition along a trajectory of paths of free passage.

Thus, the non-dissipative diffusion in classical systems in the conditions mentioned in the beginning of the section is described by the Nelson formalism, equivalent to the formalism of quantum mechanics.

\section{Examples of non-dissipative diffusion in classical systems}

\subsection{Diffusion of light gas in heavy gas}

An elementary, although trivial, example of a non-dissipative classical system is the model of ideal gas. In this model:

(1) atoms moves between collisions along classical trajectories;

(2) Collisions of an atom with others are elastic and its mean kinetic energy is conserved.

A non-trivial realization of a non-dissipative classical system with new effects is a mixture of two ideal one-atomic gases when masses of two kind atoms sufficiently differ and concentration of the light component is small. At diffusion of light gas in heavy we assume that to above mentioned conditions (1) and (2) of ideality of gases the third requirement is added also about that:

(3) the light atoms of mass $m$ collide mainly with the heavy atoms of mass $M$ and at $m / M \ll 1$ recoil is very small.

The kinetic theory of dissipative diffusion of light gas in the heavy has been developed by Lorentz [1] at the assumptions that the mixture is in thermodynamic equilibrium when velocity of light atoms is larger than heavy atoms and the last ones can be taken as rested. At these simplifications it is possible to calculate transport coefficients, including diffusion constant.

For our purposes is not necessarily to neglect by random changing of energies of particles since it is enough lack of dissipation when energy exchange of diffusing particles with medium is balanced. Although, there the evaluation of diffusion constant becomes complicated, but it is possible to use results of purely coordinate description of the previous part of the paper for prediction of new properties and effects in the system, while the diffusion constant can be considered as a parameter estimated from the theory of gases and improved by observations. 
Thus, in this system an initial energy of light atoms is conserved throughout large enough number of collisions $n_{D} \sim M / m$, corrections on a low dissipation are small and of order $\delta E \sim m / M$. At lack of external forces a path of free passage of the light particle $n_{D} l_{D}\left(l_{D}\right.$ - the free pass length) during the time $t_{D}$ is a region of nondissipativity in the first approximation.

The mean energy of the external force transferred to the particle between collisions, also is conserved with except a small part utilized for the compensation of dissipation along the direction of acceleration. In a general case it is enough to assume a capability of a full compensation of the mean loss on dissipation in the direction of initial momentum of the particle by means the external field and in the such effectively non-dissipative systems qualitative new properties the nondissipative diffusion will be exhibited in a pure form in full volume of the system.

Ordinary phenomena at diffusion of light gases in a heavy at low pressure when a free pass length is comparable with size of a container are well-known [1]. So, we will consider in these conditions only essentially new effects of nondissipativity.

In ordinary gases with heavy molecules at diffusion of atoms of light elements a region non-dissipativity is of order $L_{N D} \sim\left(10^{2} \div 10^{3}\right) l_{D}$ and $t_{N D} \sim L_{N D} / \bar{v}$, where $\bar{v}$ - mean square velocity of a light atom. These sizes and times are small for gases in a normal state, but for dilute gases the sizes are comparable to sizes of a container and times are sufficient for passing by the light atoms the same distance.

At thermodynamic equilibrium in the laboratory frame fluctuation of a value of velocity of light atoms is the same as a mean velocity of heavy gas molecules. Therefore fluctuation of energy of the light atoms is of order:

$$
\delta E \simeq \frac{1}{2} m \overline{V^{2}}=\frac{m}{M} \frac{1}{2} M \overline{V^{2}}=\frac{m}{M} \frac{3}{2} k T .
$$

Since at non-dissipative diffusion:

1) mean energy and mean drift velocity of light atoms and also the drift acceleration are defined mainly by an external field, the thin flat layer of light gas can enter in a volume with heavy gas from one partition wall and escape it (with particular losses) from other partition wall by means of the diffusion mechanism (without formation of flows in the medium);

2) in time of non-dissipativity the light gas has not time to come to full temperature balance with heavy gas, thus a dependence of a diffusional flow on temperature of the medium is sufficiently weaker, than at usual equilibrium requirements;

3) ordinary properties of a diffusional flow of a light gas in heavy are related mainly with a vacuity of a low temperature medium (low pressure or low density) effects, while the non-dissipativity effects can be realized even at high enough temperatures and densities the medium;

4) some known phenomena, specific to gases at very low pressures, can be realized at more high pressures also with a pressure "growing" coefficient of order $\sim M / m$ 
5) there should be added the probability amplitudes and in concentration distribution and diffusion flow of light atoms the wave properties (quasi-quantum effects) will be exhibited determined by the diffusion constant;

More detail discussion of these effects will be presented below and in forthcoming publications.

\subsection{Non-dissipative diffusion of neutrons and light atoms}

Requirements for realization of non-dissipative diffusion of neutrons in condensed media of heavy nuclei are, on the one hand, more favorable, than for diffusion of atoms, since the scattering happens on the nuclei. Because of small cross-section of nuclei and neutrons the free length should be large only in comparison with sizes of nuclei that is satisfied almost always, except neutron stars only.

On the other hand, for particular matter with heavy enough nuclei it is necessary to find a region of energies of diffusing neutrons when ionisational loss is insufficient and a quantum-mechanical wavelength is small enough, so that neutrons can be considered as classical particles. Number of collisions $N_{(1)} \sim M_{(1)} / m_{n} \sim 10^{2} \div 10^{3}$ defines a region non-dissipativity when recoil at neutron's scattering on a nucleus is transferred only to the nucleus.

If recoil is transferred to a molecule or a cluster with $k$ nuclei with total mass $M_{(k)}$, a number of collisions in the region non-dissipativity can be much more: $N_{(k)} \sim M_{(k)} / m_{n} \sim 10^{3} \div 10^{2 k}$

At last, when recoil at neutron's scattering is transferred to whole crystal of total mass $M$, a region of non-dissipativity practically will coincide with crystal sizes and it is possible to consider the collisions of neutrons as elastic with very high accuracy. This is an effect inverse to the Mössbauer effect.

Thus, the new effects of non-dissipative diffusion of baryons and light nuclei are:

(1) capabilities to drive flows of neutral particles by regulating a composition, density and temperature of the medium;

(2) occurrence of an effect inverse to the Mössbauer effect with a macroscopic region of non-dissipativity;

(3) in the medium from heavy elements it is possible to study possibilities of delivery of baryons and impurities of light elements into a definite region with a given distribution.

In the considered systems simplicity of realization of conditions for nondissipativity is compensated by essentially smaller sizes and intervals of time of nondissipativity region in comparison with diffusion of atoms. Nevertheless, the fact of the existence of such region can be used for many technological purposes operating with distances of molecular scales.

\subsection{Non-dissipative diffusion of electrons and ions}

In a weak ionized gas concentrations of electrons and ions are small and a number of collisions of electrons with each other and with ions is much less than number of collisions of electrons with neutral molecules. If mean energy of electrons, including the energy gained in an external field, is insufficient for excitation or 
ionization of molecules, the collisions of electrons with molecules in first approximation are elastic [1].

In addition, due to smallness of ratio of masses of electron $m_{\mathrm{e}}$ and molecules $M$, at collisions electron's energy changes insufficiently and we can neglect by velocity of molecules.

At ordinary dissipative diffusion external fields lead to motion of a free charge with velocity proportional to external force with a mobility constant and acceleration of the charged particle is insufficient. At non-dissipative diffusion motion of electrons in external electrical and magnetic fields is described by the equation of motion where a mean drift acceleration $\mathbf{a}$ is:

$$
m \mathbf{a}=e \mathbf{E}+\frac{e}{c}[\mathbf{v H}] .
$$

As a result, effects non-dissipativity will be exhibited in the form, that:

(1) flow of accelerated electrons in the electric field and a current strength increase on an exit because of higher drift velocity of electrons;

(2) in magnetic field the effects leading to more and more increasing circular velocity will be exhibited;

(3) particular interest will be open in possibilities of theoretical modeling of low dissipative conductivity processes in various media which are close to hightemperature superconductivity, since here former limitations will be partially removed and essentially new effects can be revealed.

\section{Analogues of quantum effects in classical systems \\ 3.1. Effects of an interference in classical systems}

At non-dissipative diffusion the probability amplitudes addition law and the superposition principle take place which firstly have been discovered in quantum systems. As it will becomes clear, this property is characteristic for all systems with non-dissipative diffusion.

For this reason, in the classical systems, considered in the first section, consequences of the probability amplitudes addition law, in particular, interference effects for a probability distribution, will appear also.

At diffusion of light classical particles in a medium of heavy ones in a rectangular box we can consider ensemble of the light particles which having started to diffuse with the left wall of the volume with constant drift velocity $v_{x}$ along $x$ axis, at given time have reached the right wall.

Further we will consider a standard two slit interference experiment, but dealing with classically moving particles, quantum mechanical wave length of which is much less than a characteristic size of the slits, and instead of the Planck constant there figures a diffusion constant $\hbar \rightarrow 2 m v$. The interference picture will correspond thus to a probability wave with a wave length:

$$
\lambda=2 \pi \cdot \frac{2 m v}{p_{x}}=2 \pi \frac{\Gamma_{D}}{p_{x}} .
$$

On the first and second series of experiments we will place in the middle of the volume a partition wall with a narrow slit, at first, above the wall's middle and then below the middle. Then in distribution of the passing light atoms, registered on the 
right wall, there will be maximums opposite to that from two slits which has been open.

At last, on the third series of experiments we will dispose the partition wall with two open slits. Then, if diffusion of light particles in the given medium is adequately close to non-dissipative, the interference picture in the probability distribution of the passing particles registered on the right wall will be observed.

The wave behavior of ensemble of classically moving light particles on this experiment obviously demonstrates qualitative difference of non-dissipative diffusion of light particles in medium from heavy ones from usual dissipative diffusion of heavy particles in a medium of light ones. Notice, that as ensemble of light particles there must be understand multiple repeated diffusion of one kind of particles in the given region with heavy gas, and here the interference picture is the result of addition in this ensemble of probability amplitudes of two alternatives of transition through two slits.

\subsection{Discrete energy levels of oscillator and the uncertainty relation}

Let us consider in the same volume non-dissipative diffusion of a light charged particle at the presence of an external electromagnetic field, so that the particle make transverse harmonic oscillations around a central horizontal axis of the box. Since this diffusion process is described by the Schrödinger equation, the energy levels of such oscillations will be discrete:

$$
E_{n}=2 m v \omega(n+1 / 2) \text {. }
$$

It will be possible to measure experimentally the discrete mean energy of particles at vertical oscillations in addition to continuous energy of horizontal drift. It can be registered, for example, on the Doppler line broadening of radiation of oscillating light ions. Maximums of vertical deviations will be approximately discrete also.

The presence of the "zero-point energy» of oscillations in this case expresses non-localizability of the ensemble of diffusing classical particles due to the diffusional uncertainty relations (19).

At first sight it seems paradoxical since the trajectories of light particles have classical parts between collisions where their coordinates and momenta can be defined "precisely" (at comparison with a free length and a mean momentum). However, the ensemble of such particles is characterized by a deviation of trajectories and a probability amplitude with a particular wave vector. Therefore, at localization of the ensemble of diffusing particles in a finite region, mean square deviations of coordinates and momenta in the ensemble appear related by the uncertainty relation. Then a localization region of the ensemble will small, then it will be high uncertainty of momentum.

Thus, the measuring of lowest energy level of harmonic oscillations in this experiment can be considered as confirmation of the uncertainty relation for nondissipative diffusion in classical systems.

\subsection{Discrete energy levels and discrete angular momenta of classical systems}

Construction of a macroscopic analogues of quantum systems at diffusion of a light classical particle in an external field where energy levels and an angular 
momentum are discrete, appears now related only with selection of medium of heavy particles, diffusing light particles and corresponding configuration of external fields.

In particular, the angular momentum discreteness will appear in "filling" of levels of hydrogen-like "quasiatom" at dissipation of "test" diffusing particles on these structures and also at the presence of magnetic field.

Macroscopic analogues of hydrogen-like and more complex "quasiatoms" and also discreteness of levels in magnetic field can appear useful both in technological problems and at visual demonstrating of earlier difficult explainable aspects of quantum mechanics.

\subsection{Quantum statistics in classical systems}

Above we considered an ensemble of light particles, assuming that any light particle collides only with single heavy particle of medium, i.e. it is a one-particle problem. Let us consider multi-particle problems where concentration of light particles is not small, collisions between them are essential and we come to the problem about ideal gas of light particles making non-dissipative diffusion. Since at collisions of light particles with each other their energy redistributes, these light particles come to thermodynamic equilibrium among themselves promptly, than with the medium.

Earlier such problem was considered at electron's diffusion in medium of neutral atoms or ions [1]. There a temperature $T_{e}$ of electronic gas sufficiently higher than medium's temperature $T_{i}$.

For our purposes it is interesting the fact that gas of light particles in medium of the heavy will be described by quantum statistics where the role of the Planck constant plays a diffusion constant $\hbar \rightarrow 2 m v$. Manifestations of effects of quantum statistics will be numerous and for this reason we consider some of them only which demonstrate main properties of quantum statistics.

The first property is indistinguishability. If in multiparticle system energy levels have equal probability and particles are assumed distinguishable, we come to the Boltzman distribution:

$$
n=n_{0} e^{-E_{n} / k T}
$$

If levels have equal probability also, but particles are indistinguishable, the BoseEinstein distribution will be obtained.

$$
n=\frac{n_{0}}{e^{E_{n} / k T}-1} .
$$

Therefore there is a question how in system of classical distinguishable particles can appear quantum statistics?

The solution to this problem has been discovered by Bayer and Terzof [7] and consists in that the assumption about equal probability of levels appears too strong limitation. In fact, as Bose did from the beginning, there is enough an assumption that sum of probabilities of all alternative ways of fillings is equal to unity and that system's total energy, obviously, does not change at all possible fillings:

$$
E=\sum_{n} N_{n} E_{n} .
$$


At such general assumptions, as it has appeared, in gas from distinguishable particles the Bose-Einstein distribution generally takes place and only at the additional equal probability requirement it is narrowed down to the Boltzmann distribution [7]. Thus, the indistinguishability of particles in the quantum statistics appears effectively, being a consequence of certain limitations for system from distinguishable particles.

The second property of quantum statistics which we will consider is an analogue of the Bose-Einstein condensation for light gas at non-dissipative diffusion in heavy gas. This effect can be observed by creating conditions similar to usual quantum effect, but with replacement $\hbar \rightarrow 2 m v$. It means that a quasi-Bose-Einstein condensation can be realized on macroscopic systems also, by choosing a content of a medium and a diffusing light gas.

The third in essence new property is the Pauli's exclusion principle for particle systems which are described by wave functions, antisymmetric at transpositions of particles. Still neglecting details of connection of spin and statistics, here we notice only that the theory of non-dissipative diffusion generally admits such states irrespective of the reasons of their origin. Therefore, if we create requirements where there is diffusion with such wave functions, then in classical systems we can observe consequences of the Fermi-Dirac statistics and Pauli's exclusion principle.

\section{Quantum mechanics as the theory of non-dissipative diffusion}

\subsection{Insufficiency of standard interpretations of quantum mechanics}

Quantum mechanics is a theoretical basis of a modern physics and reliability of its mathematical formalism (in various formulations) for description of physical phenomena is beyond doubt.

However at concerning a physical interpretation of quantization the situation is opposite and there is common consent that in that aspect quantum mechanics is far of to be finished. The standard interpretations of quantum mechanics are based on in fact ideological statement that we should divide the reality on macro- and microscopic objects and for the last ones should restrict classical mechanics and to add something new. In this sense they are in fact "revolutionary" approaches. However there are many questions, on which they do not answer, postulating the necessary answer, or giving inconsistent answers:

(1) Why quantization is necessary?

(2) Why there are quantum fluctuations and what they mean?

(3) What in fact fluctuates: a background, acting on a particle, or the particle in itself at a smooth background?

(4) Why there should be added probability amplitudes of alternative events, instead of probabilities?

(5) What principally distinguishes quantum particles from the classical ones?

(6) If there are large fluctuations of energy and momentum of a particle, what is a source of these large energy and momentum?

(7) Is such violation of energy and momentum conservation compensated by decreasing of energy and momentum of something?

(8) Are fluctuations related to structure of spacetime or not?

Existence or lack of answers to these questions practically does not influences to efficiency of applications of quantum mechanics and thus such searches did not represented a particular interest. However, from the point of view of 
prospects of further development of quantum theory the correct answers are necessary and important.

The stochastic mechanics, in which quantum fluctuations are reduced to a non-dissipative diffusion, in that sense represents a sufficient progress. This version of quantum mechanics is a single one free from "ideological" partitioning of reality to macro- and micro-objects.

The next step was consisted in constructing a microscopic theory of such diffusion in the case of quantum fluctuations, which will be considered below.

\subsection{Non-dissipative diffusion in quantum systems}

A general case of Markovian processes with symmetric on time directions equations for transition probabilities forward and back on time has been considered still by Kolmogorov. Then first example of non-dissipative diffusion has been found at attempts of stochastic treatment of quantum mechanics. At first Fenyés [2] has related quantum fluctuations with non-dissipative diffusion processes and Nelson $[3,4]$ formulated mathematically consistent kinematics and dynamics of such diffusion from which quantum mechanics formalism directly followed.

The Schrödinger equation for a quantum particle, at first, differs from a diffusion equation only by the presence of imaginary unity and secondly, describes temporary evolution of complex amplitude of probability, being system from two equations for real and imaginary parts of this amplitude. Besides, unlike usual diffusion, quantum mechanical equations are reversible in time, mean kinetic energy of a free particle is conserved and a mean acceleration of a particle by an external force is defined by Newton's laws.

For this reason there appeared an idea to describe quantum fluctuations of particles as conservative diffusion when energy exchanging between diffusing particle and vacuum is balanced due to some reasons. Then there is symmetry under the time reversal and two diffusion equations for forward and backward in time transition probabilities, together with Newton's law, lead to one a complex Schrödinger equation for a complex probability amplitude.

Nelson at first has formulated kinematics for the symmetric on time Brownian motion which has been explained in the previous part of the present paper. From it follows, particularly, the uncertainty relation (19). Further, assuming validity of the Newtonian dynamics, from the diffusion equations for flows (20) and (22) it has obtained the equation for the probability amplitude (26):

$$
i(2 m v) \frac{\partial \psi}{\partial t}=H \psi,
$$

which turns to the Schrödinger equation for the diffusion constant $v=\hbar / 2 m$, where $\hbar$ is the Planck constant.

Thereby it has been proved that the formalism of quantum mechanics is equivalent to the formalism of non-dissipative Brownian motion of classical particles in the physical vacuum.

However, the kinematics for a diffusing particle, which was used by Nelson for obtaining the equations of quantum mechanics, takes place only for the EinsteinSmoluchowski type diffusion when friction is very large and it promptly balances any external force. At the same time, the dynamical assumptions (21) and (23), 
necessary for derivation of the Schrödinger equation, take place only for diffusion with very small friction, describing by the Langevin-Ornstein-Uhlenbeck theory.

Nelson, who understood incompatibility of such requirements for ordinary diffusion in classical systems, considered these assumptions, before clearing up of their physical sense, as postulates for treatment of quantum fluctuations. However, the idea of description of quantum phenomena in terms of the classical random processes, let even specific, supposes a capability of realization of such fluctuations in classical systems at least in principle. If in a classical case it leads to interior inconsistencies, all approach also becomes doubtful and inconsistent.

However, in the previous sections it has been shown that, at first, nondissipative diffusion can be realized in classical systems, but under the conditions different from conditions of ordinary Brownian motion and, secondly, not only Nelson's kinematics, but also its dynamical assumptions also naturally follow from the theory of diffusion in such systems.

\subsection{Stochastic mechanics as a physical basis of quantum mechanics}

In contrast to the standard revolutionary interpretations, the stochastic mechanics is based on a careful and conservative point of view and naturally answers to all those problems which seemed as mysterious for the former interpretations. In stochastic mechanics there no special quantum objects and it is based on the physical fact that at motion in the physical vacuum classical particles possesses specific fluctuations inversely proportional to their mass.

Thus, the vacuum properties are assumed such that at interaction with it:

(1) spatial coordinates of a particle fluctuate and the particle makes a specific Brownian motion;

(2) the equations describing the Brownian motion of the particle are reversible in time that further leads to conservation of mean energy of the particle (nondissipative or conservative diffusion);

(3) a diffusion constant $2 v$ is inversely proportional to particle's mass: $2 v=a / m$, where the constant $a$ follows from coincidence with quantum mechanics and it appears equal to $a=\hbar$.

In stochastic mechanics the answers to the problems 1-3 of the previous section are given the same as in any system of interacting particles and fields, i.e. by the fact of the existence of a media with particular properties.

But to key and most mysterious for all previous treatments problems 4-5 it has been given very clear answer by Nelson $[3,4]$. It has shown that from the nonlinear equations of conservative diffusion, relating a probability density and transition velocities of classical particles, there follow the linear Schrödinger equations for a complex probability amplitude.

The large fluctuations of energy of a particle happen due to interaction with the physical vacuum and, consequently, at any moment growing of energy of the particle is compensated by corresponding local decreasing of energy of the physical vacuum and on the contrary. It is the answer on 6th and 7th problems. The such answer to 7-8th problems in stochastic mechanics from the physical point of view has been considered in the paper [6] where the diffusional treatment of gravitation has been proposed.

The fact that growing of particle's energy at a quantum fluctuation should lead to decreasing of energy of the physical vacuum to the same value has direct 
observable consequences. At large concentration of particles in small region of space the energy of vacuum will be reduced at any moment and mean energy of vacuum here will be lower than at distant regions without particles. Due to decreasing of "reserves" of vacuum energy the intensity of fluctuations of particles will be reduced that will be exhibited as decreasing of the diffusion constant. It leads to slowing down of rate of all quantum processes, thus frequencies will be redshifted, proper times will be dilated and particles from regions of normal fluctuations will slowly drift to this region of dilated fluctuations. All these are the characteristic properties of gravitation which in the paper [6] is treated as inhomogeneity of quantum fluctuations. More detail discussion of such diffusional treatment of gravitation will be presented in forthcoming publications.

\section{Conclusion}

Thus, in low-dissipative systems always there is a region of non-dissipativity where throughout large number of collisions it is possible to neglect by dissipation. A model of such non-dissipative diffusion is diffusion of a light particle in a dilute medium of heavy particles. In the non-dissipativity region of such system a mean energy of the light particle is conserved and the diffusion process is described by more general theory with equations symmetric under both directions of time. Thus, there is the elementary phase volume $\Gamma_{D}=p_{D} l_{D}$ of paths of free passage of the light particle and a diffusion constant then is equal to $v=\Gamma_{D} / 2 \mathrm{~m}$.

Main difference of the theory of non-dissipative diffusion from the usual consists that in this theory there should be added probability amplitudes instead of probabilities. There holds a superposition principle for these amplitudes since nonlinear diffusion equations for forward and backward in time transitions are linearized, turning to the complex Schrödinger equation. There are also the uncertainty relations for uncertainties of coordinates and momenta in the ensemble of particles, restricting product of their variances by the elementary phase volume.

Thus, it has appeared that the formalism of quantum mechanics has wider area of applicability than especially quantum systems and describes in fact a classical non-dissipative diffusion with a homogeneous diffusion constant, realized at diffusion of light particles in dilute medium of heavy particles. Quantum mechanics is only a particular case of such diffusion with the elementary phase volume $\Gamma_{D}=\hbar$ and, therefore, with the diffusion constant $v=\hbar / 2 m$. Examples of classical systems with various diffusion coefficients show that the effects non-dissipativity are nontrivial, enough universal and represent a new area of research with observable consequences and many possible applications.

\section{Appendix. Dissipative diffusion and the Brownian motion}

Microscopic bodies consisting of a large number of molecules, in fluids and gases in general case make a dissipative Brownian motion. The such Brownian particle should be small enough for exhibiting fluctuations of molecular motion in the medium, but also should be large enough to be observed. Therefore it is sufficiently larger than molecules, in a dense medium at each moment a large number of molecules collide with it and a friction is essential. 
In the dense medium, in particular, in fluids where friction is large, action on the particle of external force is balanced by frictional force and the Brownian motion in such medium is described by the Einstein-Smoluchowski theory [4]. Thus, particle's additional energy, gained from the external force, transfers into heat, acceleration disappears and the Brownian particle makes only a random motion with a mean drift velocity proportional to the external force and the mobility. Since a relaxation time of velocity of the particle is not enough in comparison with time intervals $\Delta t$, when particle's positions are fixed, finite shifts of the particle become independent and the particle's trajectory is non-differentiable on time.

In a dilute medium with a small friction, for example, in ideal gases, coordinates of Brownian particles are differentiable on time, but velocity has a random component due to acting of random forces of the medium. The external forces can exceed frictional force and can accelerate the particle that is described by the Langevin-Ornstein-Uhlenbeck theory [4].

For the description of the Brownian motion of a particle in the dense medium described by the Einstein-Smoluchowski theory, we measure its coordinates through small, but finite time intervals $\Delta t$, and particle's motion between points $\mathbf{x}(t-\Delta t)$, $\mathbf{x}(t)$ and $\mathbf{x}(t+\Delta t)$ approximate by smooth classical trajectories. Velocity $\mathbf{b}(x, t)$ of particle's drift is defined on these artificially smoothed parts of a trajectory by averaging on all trajectories which are going out given event $\mathbf{x}(t)$ and turn to limit $\Delta t \rightarrow 0$ only after the averaging:

$$
\mathbf{b}_{+}(\mathbf{x}(t), t)=D_{+} \mathbf{x}(t) \equiv \lim _{\Delta t \rightarrow 0+} E_{t} \frac{\mathbf{x}(t+\Delta t)-\mathbf{x}(t)}{\Delta t}
$$

where $E_{t}$ - the conditional probability and $D_{+} \mathbf{x}(t)$ is the such averaged time derivative.

Particle's shifts then are described by the stochastic equations:

$$
d \mathbf{x}_{+}(t)=\mathbf{b}_{+}(\mathbf{x}(t), t) d t+d \mathbf{w}_{+}(t),
$$

where $d \mathbf{w}_{+}(t)$ is a random process with properties (8). Thus, in this approximation the external forces are balanced by frictional force and lead only to the drift velocity $\mathbf{b}_{+}$, proportional to the external field: $\mathbf{b}_{+}=b \mathbf{F}$, where $b$ is the mobility. Strong external fields, sufficiently exceeding frictional force, lead to the local heating of the fluid and to hydrodynamic effects which are already beyond the diffusional approximation.

Probability density $\rho(x, t)$ is normalized as in (9) and satisfies the equation:

$$
\partial \rho / \partial t=-\nabla \cdot\left(\mathbf{b}_{+} \rho\right)+v \Delta \rho,
$$

and corresponding transition probabilities, defined as:

$$
\rho\left(x^{\prime}\left(t^{\prime}\right), t^{\prime}\right)=\int p_{+}\left[x^{\prime}\left(t^{\prime}\right), t^{\prime} ; x(t), t\right] \rho(x(t), t) d x(t), \quad\left(t^{\prime}>t\right),
$$

satisfy the equation $\left(t^{\prime \prime}>t^{\prime}>t\right)$ :

$$
p_{+}\left[x\left(t^{\prime \prime}\right), t^{\prime \prime} ; x(t), t\right]=\int p_{+}\left[x\left(t^{\prime \prime}\right), t^{\prime \prime} ; x\left(t^{\prime}\right), t^{\prime}\right] p_{+}\left[x\left(t^{\prime}\right), t^{\prime} ; x(t), t\right] d x\left(t^{\prime}\right) \text {. }
$$

Mean time derivatives from functions of coordinates and time $f(x, t)$, according to (38) and (39), are defined as: 


$$
D_{+} f(x(t), t)=\left(\partial / \partial t+\mathbf{b}_{+} \cdot \nabla+v \Delta\right) f(\mathbf{x}(t), t) .
$$

In a medium with weak friction the coordinates of the Brownian particle are differentiable on time, while the velocities contain both regular and random contributions. In the theory of such diffusion, formulated by Langevin, Ornstein and Uhlenbeck, the equations of motion look like:

$$
\begin{aligned}
d \mathbf{x}(t) & =\mathbf{v}(t) d t, \\
\Delta \mathbf{v}(t) & =-\beta \mathbf{v}(t) \Delta t+\frac{1}{m} \mathbf{F}(\mathbf{x}(t)) \Delta t+\Delta \mathbf{B}(t),
\end{aligned}
$$

where $\mathbf{B}(t)$ has properties:

$$
\begin{aligned}
& \lim _{\Delta t \rightarrow 0} E_{t}[\Delta \mathbf{B}(t)]=0, \\
& \lim _{\Delta t \rightarrow 0} E_{t}\left[\Delta B_{i}(t) \Delta B_{j}(t)\right]=\frac{6 \beta k T}{m} \delta_{i j} d t .
\end{aligned}
$$

Because of weakness of frictional force, the external forces can exceed it and a mean drift acceleration $\mathbf{a}$ is related to the external force $\mathbf{F}$ and frictional force by Newton's laws:

$$
\mathbf{a}=\lim _{\Delta t \rightarrow 0} E_{t}\left[\frac{\Delta \mathbf{v}(t)}{\Delta t}\right]=-\beta \mathbf{v}(t)+\frac{1}{m} \mathbf{F}(\mathbf{x}(t)) .
$$

At a weak external field frictional force again exceeds the external forces, the mean acceleration disappears and we return to the Einstein-Smoluchowski theory assuming the such equilibrium.

Thus, depending on a relation of frictional and external forces, two kinds of dissipative diffusion can be realized. If frictional force is larger or external forces are weak, then the coordinates of the Brownian particle are stochastic and the external force field does not accelerate it (Einstein-Smoluchowski theory). If friction is small, while the external force is strong enough, the coordinates change smoothly, but the particle's velocity has a stochastic component and the drift acceleration is defined by Newton's law (the Langevin-Ornstein-Uhlenbeck theory).

\section{References}

1. Landau L.D., Lifshitz E.M., Physical Kinetics, Pergamon Pr., 1981.

2. Fényes I. (1952) Z. Phys., 132, 81.

3. Nelson E. (1966) Phys. Rev., 150, 1057.

4. Nelson E. (1985) Quantum Fluctuations. Princeton. U. Press.

5. Zakir Z. (1987) A generalized principle of relativity as a foundation for the theory of stochastic space and time. Prepr. PTI-43-87-FVE, Tashkent 1987; arXiv:hep-th/9901013.

6. Zakir Z. (1999) Gravitation as a Quantum Diffusion. arXiv:hep-th/9812254; arXiv:gr-qc/9906079.

7. Tersoff J., Bayer D. (1983) Phys. Rev. Lett. 50, 8, 553. 